\title{
Virulence Characteristics and Molecular Epidemiology of Pyogenic Liver Abscess Causing Multidrug Resistant Klebsiella pneumoniae in Wenzhou, China
}

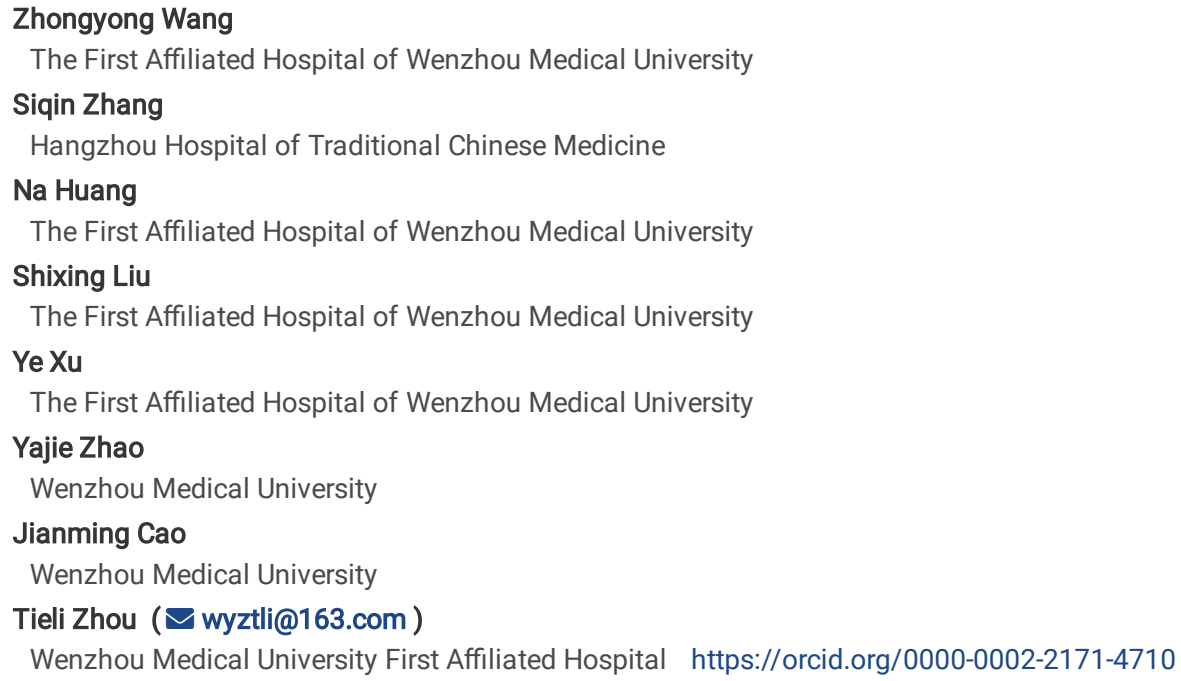

Research article

Keywords: pyogenic liver abscess, multidrug resistance, Klebsiella pneumoniae, virulence characteristics, molecular epidemiology

Posted Date: January 21st, 2021

DOI: https://doi.org/10.21203/rs.3.rs-42660/v3

License: (c) (i) This work is licensed under a Creative Commons Attribution 4.0 International License. Read Full License 


\section{Abstract}

Background: To date, little is known about the virulence characteristics of pyogenic liver abscess (PLA) that cause multidrug resistant (MDR) Klebsiella pneumoniae (K. pneumoniae), and this might be due to that these strains are rare. This study aimed to analyze the virulence characteristics and molecular epidemiology of 12 MDR strains obtained from 163 PLA cases in a tertiary teaching hospital from the perspective of clinical characteristics, virulence phenotypes, and genotypes.

Results: The results of growth curves, string test, capsular quantification, serum killing test, biofilm formation assay, and infection model revealed that the virulence phenotypes of the 12 PLA-causing MDR K. pneumoniae were similar or even more obvious than those of typical hypervirulent $K$. pneumoniae strains. These MDR strains were mainly non-K1/K2 serotypes and carried multiple virulence genes. The results of multilocus sequence typing (MLST) illustrated that the MDR strains were categorized into 9 sequence types.

Conclusions: This study is the first to analyze the virulence characteristics in PLA-causing MDR strains. Our data exhibited the coexistence of hypervirulence and MDR in PLA-causing MDR K. pneumoniae strains, and the clones of those PLA-causing MDR strains were diverse and scattered. This study first found one ST11 carbapenem-resistant hypervirulent strain in PLA.

\section{Background}

Klebsiella pneumoniae is a major Gram-negative bacterium that is responsible for urinary tract infections, pneumonia, bacteremia, and intra-abdominal infections worldwide [1-2]. In the past three decades, $K$. pneumoniae is divided into two non-overlapping populations. One is termed as classic $K$. pneumoniae (cKP), which is a pathogen that causes hospital-acquired infections in immunocompromised patients and is notorious in acquiring antimicrobial resistance, while the other is hypervirulent K. pneumoniae (hvKP) [1-3]. In contrast to cKP, an emerging variant that was first reported in Taiwan in 1986 is hypervirulent in causing severe invasive community-acquired infections and disseminates infections among immunocompetent individuals. According to recent studies, hvKP exhibits hypervirulent phenotypes and genotypes, and is susceptible to conventional antimicrobial agents except for the intrinsic resistance to ampicillin [1-3].

Pyogenic liver abscess (PLA) is a potentially life-threatening suppurative infection of hepatic parenchyma that occurs worldwide [2,4,5]. Klebsiella pneumoniae has been emerged as a predominant pathogen of PLA across Asian and European countries, as well as the United States. There is no denying that K. pneumoniaeinduced pyogenic liver abscess (KP-PLA) is a serious clinical challenge due to its association with mortality [5-6]. Hypervirulent $K$. pneumoniaeinduced PLA usually occurs in young and healthy community individuals with no identified source of infection, and the cryptogenic abscess migrates to distant sites, leading to extrahepatic complications, such as endophthalmitis, meningitis, and necrotizing fasciitis [2,5,7]. Most of the isolates from KP-PLA are susceptible to majority of antibiotics, and the antibiotic resistance rates are less than $10 \%$ [8]. Antibiotic resistance is a phenomenon that is primarily associated with cKP. Interestingly, most of the recent reports have revealed the convergence of virulence and resistance in $K$. pneumoniae, and most of these phenomena were commonly caused by plasmid mediated resistance traits and virulence genes transfer [9-10]. In our previous study [11], 12 multidrug resistant (MDR) K. pneumoniae were isolated from non-cryptogenic PLA, which are considered as cKP. Although KP-PLA caused by antibioticsusceptible hypervirulent strains has been well reported, MDR K. pneumoniae isolates from KP-PLA are rare and have not been well identified yet, particularly with regard to virulence characteristics and molecular epidemiology $[2,7,12]$. Whether these MDR $K$. pneumoniae isolates were indeed traditional cKP or combined with hypervirulence still remains to be unknown. Since the virulence of $K$. pneumoniae can assist the pathogen to resist host innate immunity and infect the host invasively with high pathogenicity $[1,13]$, the convergence of virulence and resistance of $K$. pneumoniae will pose more challenges in treating KP-PLA. In addition, hypervirulent strains usually possesses thick capsular polysaccharide, anti-serum capacity and multiple virulence factors (hypermucoviscosity, capsular serotype, virulence genes, related clones and so on) $[14,15]$. Therefore, it is necessary to gain knowledge with regard to virulence characteristics and molecular epidemiology in the PLA-causing MDR K. pneumoniae urgently.

Hence, in this study, the virulence characteristics and molecular epidemiology of PLA-causing MDR $K$. pneumoniae were investigated by collecting the strains for over a 2-year period from KP-PLA patients in a tertiary teaching hospital to provide significant insights in the development of effective therapeutic strategies for KP-PLA.

\section{Results}

\section{Antimicrobial susceptibility testing and growth curves}

\section{String test and quantification of capsule}

The string test and quantification of capsule were performed to evaluate the hypermucoviscosity and the capsule of PLA-causing MDR strains. The results of string test revealed that two (2/12, 16.6\%) MDR strains (FK4176 and FK4737) had hypermucoviscosity, while five (5/12, 41.7\%) strains (FK3645, FK3736, FK3914, FK3953, and FK3992) had hypermucoviscosity among the typical hypervirulent strains. Quantification of the capsule further revealed that the capsular polysaccharide content of MDR strains was significantly lower than that of typical hypervirulent strains, but was significantly higher than that of ATCC 700603, and the differences were statistically significant $(P<0.05)$, (Figure 2).

\section{Biofilm formation assay}

In addition to measuring the capsule, biofilm formation assay was also conducted. The OD values of the biofilms formed by MDR strains ranged from 0.31 to 0.80 , with an average value of $0.58 \pm 0.19$; and the $\mathrm{OD}$ values of the biofilms formed by typical hypervirulent strains ranged from 0.06 to 0.39 , with an 
average value of $0.27 \pm 0.10$. The biofilm formation ability of MDR strains was significantly higher than that of typical hypervirulent strains $(P<0.05)$, (Figure 3).

\section{Serum killing test}

To evaluate the sensitivity of the PLA-causing MDR strains to serum, the serum killing test was performed. All MDR strains and typical hypervirulent strains isolated from KP-PLA were found to be susceptible to serum, and there were no significant differences between the two groups $(P>0.05)$, (Figure 4$)$.

\section{Infection model of Galleria mellonella larvae}

To further verify the pathogenicity of the PLA-causing MDR strains, an in vitro model of G. mellonella larvae was constructed. The mortality of the larvae depended on the inoculum concentration and action time of the three MDR strains and three typical hypervirulent strains $(P<0.05)(F i g u r e 5 A, B, C, D, E, F)$. In addition, the lethality of MDR strains and typical hypervirulent strains was similar when using $10^{6} \mathrm{CFU} / \mathrm{mL}$ bacterial suspensions to infect the larvae, but both were significantly higher than that of the standard strains ATCC 700603 and PBS controls $(P<0.05)$, (Figure 5G).

\section{Polymerase chain reaction for capsular serotypes and virulence genes}

To investigate the virulence genotypes of the PLA-causing MDR strains, capsular serotypes and virulence genes were measured. Among the 12 PLA-causing MDR strains, four were with K1 serotype (FK3228, FK3518, FK3599, and FK4737), one was with K2 serotype (FK4276), one was with K20 serotype (FK4176) and six were of non-type. Except $\operatorname{magA}(33.3 \%)$, iroN (33.3\%) and $k f u B C(50.0 \%)$, all the remaining virulence genes were present in more than half of the MDR strains (wcaG 83.3\%, ybtA 83.3\%, fimH 91.7\%, mrkD 100\%, uge $91.7 \%$, entB 100\%, and ureA 83.3\%). The prevalence of $r m p A$ and aerobactin was $83.3 \%$ and $75.0 \%$, respectively. Among the 12 typical hypervirulent strains, all were of $\mathrm{K} 1$ or $\mathrm{K} 2$ serotype, and all virulence genes were present in most of the strains (aerobactin 100\%, rmpA 100\%, fimH 100\%, mrkD 100\%, uge 100\%, ureA 100\%, entB $91.7 \%$, wcaG 83.3\%, magA $83.3 \%, k f u B C 75.0 \%$, and ybtA $75.0 \%$ ) with the exception for iroN(33.3\%). Additionally, the prevalence of numerous virulence genes in MDR strains showed no significant differences with that of typical hypervirulent strains.

\section{Multilocus sequence typing}

To conduct molecular epidemiology of the PLA-causing MDR strains, multilocus sequence typing was performed. The clones of the 12 MDR strains were diverse and scattered, and were categorized into 9 sequence types (ST23, ST11, ST29, ST65, ST86, ST320, ST367, ST420, and ST831). Among the typical hypervirulent strains, the predominant type was ST23 (8/12, 66.7\%), followed by ST65 (2/12, 16.7\%) and ST86 (2/12, 16.7\%), (Figure 6).

\section{Discussion}

As reported previously, the MDR K. pneumoniae usually causes infections in patients with underlying diseases and is considered as cKP with high resistance rate but hypovirulence $[2,12,14]$. However, $K$. pneumoniae isolates from KP-PLA has converged hypervirulence and high antibiotic resistance, which limited the clinical treatment options largely [16]. To date, little is known with regard to the virulence characteristics of PLA-causing MDR strains. Therefore, 12 MDR K. pneumoniae strains were collected from $163 \mathrm{KP}$-PLA cases and analyzed the virulence characteristics and molecular epidemiology. To the best of our knowledge, this is the first study to analyze the virulence characteristics of the PLA-causing MDR strains.

Numerous studies have reported that antibiotic resistance rates remained lower in KP-PLA [8,11,12]. Moreover, the MDR strains were rare, and patients infected with these were more likely accompanied with hepatobiliary diseases when compared to patients infected with non-MDR strains (Table S1). More importantly, the uncontrollable infections and ineffective prognosis in patients with hepatobiliary diseases might be associated with recurrent bacteremia due to MDR bacteria. This suggested that these MDR isolates might not be related to traditional cKP and acquisition of MDR might not compromise the overall virulence, requiring further verification. However, the actual virulence of these MDR strains has not been well evaluated so far.

The growth ability results suggested no fitness cost regarding the strains with resistant phenotype. In addition, the hypermucoviscosity was considered as a surrogate marker of hvKP [5]. In this study, the percentage of hypermucoviscous MDR strains was found to be slightly lower than that of typical hypervirulent strains. However, it might not be suitable to consider hypermucoviscosity as the only indicator of hypervirulence, wherein the polysaccharide capsule can protect $K$. pneumoniae from phagocytosis of immune cells and complement-mediated bactericidal action, which acts as a major virulence characteristic for hvKP [17]. The results of capsular quantification revealed that the capsular content of PLA-causing MDR strains was higher than that of the standard strain and lower than that of the typical hypervirulent strains. The standard strain ATCC 700603 that recognized as classic $K$. pneumoniae is known for producing extended-spectrum $\beta$-lactamase (ESBL) enzymes that can hydrolyze oxyimino- $\beta$-lactams, resulting in resistance to these drugs, and its virulence is less than the typical hypervirulent $K$. pneumoniae [18]. The data of capsular quantification was consistent with the data of the string test. Although the MDR strains and typical hypervirulent strains were sensitive to serum, the antiserum killing ability of these PLA-causing strains was significantly higher than that of the typical hypervirulent strains, which might be related to the content of capsular polysaccharide. Furthermore, the bacteria attaches to the surface of the host during the infectious process and are coated with polymers such as extracellular polysaccharides and DNA to form biofilms. The physical barrier formed by biofilms can protect the bacteria from phagocytes and enzymes, improving the bacterial defenses against the host and antimicrobial resistance. This meant that the biofilm formation ability of MDR strains was significantly higher than that of typical hypervirulent strains, which might be one of the reasons for the MDR strains to exhibit resistant phenotype. Moreover, G. mellonella larvae, which acts as a good model of invertebrate host infection, has been used to explore the virulence and pathogenicity of $K$. pneumoniae strains [19]. By combining with the results of virulence genes, although the siderophore genes were different in these PLA-causing MDR strains and typical hypervirulent strains, these strains still had comparable level of virulence in terms of lethality. This showed that the siderophore transport systems require further research to clarify the relationship between siderophore utilization and bacterial virulence. The 
consistency between the clinical data and the results of phenotypic assays supported the notion that the PLA-causing MDR K. pneumoniae strains were hypervirulent.

Analysis of virulence genotypes can further validate our hypothesis. K. pneumoniae strains are presented in at least 78 capsular serotypes, in which K1 and K2 are related to hvKP, and are strongly pathogenic to humans $[17,20]$. In the present study, K1 or K2 serotypes accounted for less than half of the PLAcausing MDR strains, while all the typical hypervirulent strains belonged to K1 or K2 serotypes. Although K1 or K2 serotypes can regulate the virulence of $K$. pneumoniae, hypervirulence is not unique to these capsular serotypes [21]. In addition, $r m p A$ and aerobactin are the most important genes for hypervirulence [1]. rmpA regulates the synthesis of extracellular polysaccharide capsule in order to enhance the virulence [22-23], and aerobactin is considered essential for the growth and virulence of $K$. pneumoniae by regulating iron supply [1]. In the present study, the prevalence of $r m p A$ and aerobactin in the MDR strains was slightly lower than that of typical hypervirulent strains, reflecting that the PLA-causing MDR strains might be combined with hypervirulence from the perspective of virulence genes. More importantly, wcaG, $m a g A$, and uge genes related to capsule synthesis were also prevalent in the PLA-causing MDR strains [24-25]. Additionally, the inconsistency between the results of the capsule-related genes and hypermucoviscosity supported the notion that hypermucoviscosity is not a good factor for assessing hypervirulence and should be assessed in conjunction with genotypes and other phenotypes, as well

as clinical characteristics. Moreover, the high prevalence of siderophore genes such as $y b t A$, entB, and $k f u B C$ in the PLA-causing MDR strains suggested that the ability of iron uptake might be equivalent to that of typical hypervirulent strains. Furthermore, almost all the PLA-causing MDR strains carried fimH (related to type 1 fimbriae), $m r k D$ (related to type 3 fimbriae), and ure $A$ (an a-subunit of the urease, associated with invasion) [24,26], and genetically corroborated with virulence phenotype results. The results of these genes and adherence or invasion in biofilm formation in MDR strains might explain as to why the PLA-causing MDR strains were more resistant to antibiotics than the PLA-causing typical hypervirulent strains. Therefore, clinicians should pay much attention to the MDR strains, and also carefully select appropriate management strategies to treat KP-PLA to reduce bacterial adhesion and colonization.

MLST analysis uncovered the molecular epidemiology of PLA-causing MDR strains. The clones of these MDR strains were diverse and scattered, while the clones of typical hypervirulent strains almost belonged to ST23, and this was consistent with that reported in the previous study [27]. It is commonly known that ST11-type K. pneumoniae is resistant to carbapenems, but not hypervirulent. However, the new ST11-type strain that has been emerged in recent years is simultaneously hypervirulent, multidrug resistant, and transmissible, and this could pose a serious threat to the public health [9,15]. Previous studies have revealed that ST11 carbapenem-resistant hypervirulent strains were not found in KP-PLA. To the best of our knowledge, this is the first study to describe that one ST11 carbapenem-resistant strain might be MDR-hypervirulent K. pneumoniae and has been described in KP-PLA. Importantly, further surveillance and implementation are needed to control the dissemination of infection in hospital settings and community.

\section{Conclusion}

Combining the virulence phenotypes and genotypes, the convergence of hypervirulence and multidrug resistance in PLA-causing MDR K. pneumoniae strains was observed, which might in turn lead to further emergence of "post-antibiotic" scenario. Importantly, it reminded that clinicians should be highly prudent in prescribing antibiotics to KP-PLA patients due to severe antibiotic resistance and provide inspection measures timely in view of hypervirulence-induced invasive infections, and supervisors should implement meticulous control measures to prevent such real superbug from further disseminating to patients and hospitals. Moreover, further research is needed to elucidate the mechanisms among the host, pathogen, and host-pathogen interactions. This will in turn lay a foundation to raise the awareness regarding MDR-hvKP and provide effective treatments for KP-PLA patients.

\section{Methods}

\section{Bacterial isolates, antimicrobial susceptibility testing, and growth curves}

From June 1, 2016 to December 31, 2017, a total of 163 KP-PLA cases were collected from the First Affiliated Hospital of Wenzhou Medical University (Wenzhou, China), which has an annual admission of more than 160,000 inpatients. The diagnosis of KP-PLA was conducted based on the clinical criteria $[7,28]$. Initial strains were isolated from sterile fluids (including pus, blood, and drainage fluid) of KP-PLA patients and identified as K. pneumoniae by matrixassisted laser desorption/ionization time-of-flight mass spectrometry (MALDI-TOF/MS; bioMérieux, Lyons, France). Antimicrobial susceptibility testing of $K$. pneumoniae isolates was conducted by bioMerieux VITEK-2 (BioMérieux, Marcy-l'Étoile, France) initially. MDR strains were defined as non-susceptible to three or more different antimicrobial categories [29]. A total of 12 MDR K. pneumoniae were found in $163 \mathrm{KP}-\mathrm{PLA}$ cases. Meanwhile, an equal number of antimicrobial-susceptible typical hypervirulent strains were selected as experimental hypervirulent control strains (isolated from healthy, ambulatory patients with KP-PLA and carried both aerobactin and rmpA genes) and the standard strain ATCC 700603 as the hypovirulent strain (Table 1) [1,30].

The minimum inhibitory concentrations (MICs) of ampicillin, aztreonam, ceftriaxone, ceftazidime, cefepime, imipenem, ciprofloxacin, levofloxacin, gentamicin, tobramycin, sulfamethoxazole/trimethoprim, nitrofurantoin and colistin were confirmed by agar dilution method and microdilution broth method. The results were interpreted based on the latest guidelines published by the Clinical and Laboratory Standards Institute (CLSI; Pittsburgh, PA, USA) and the European Committee on Antimicrobial Susceptibility Testing clinical breakpoints (http://www.eucast.org). Escherichia coli ATCC 25922 and Pseudomonas aeruginosa ATCC 27853 served as quality control strains. The experiment was done in triplicate.

The growth curves of 12 PLA-causing MDR K. pneumoniae isolates were measured as described by the previous methods [31]. In brief, overnight cultures of selected K. pneumoniae clinical isolates from KP-PLA and K. pneumoniae ATCC 700603 were diluted in 1:100 by Luria-Bertani (LB) broth. The cultures were incubated at $37^{\circ} \mathrm{C}$ by constantly shaking at $200 \mathrm{rpm}$. The bacterial suspensions were collected at $0,2,4,6,8,10,12,18$, and $24 \mathrm{~h}$ and the absorbance was measured at $600 \mathrm{~nm}$. Each suspension was measured in triplicate and the average of absorbance values was used for analysis. The growth of PLA-causing MDR K. pneumoniae was evaluated by plotting optical density $600 \mathrm{~nm}\left(\mathrm{OD}_{600}\right)$ values against time. The experiment was done in triplicate.

Page $4 / 16$ 


\section{String test and quantification of capsule}

The bacterial colonies of $K$. pneumoniae strain on an agar plate were scratched by an inoculation loop. The string test was considered positive when a viscous string of $>5 \mathrm{~mm}$ length was generated by the strain, and this was also considered hypermucoviscous [30].

The capsule was quantified as described previously with some modifications [10,32]. Briefly, 500 $\mu \mathrm{L}$ of cultured bacterial suspensions were resuspended and adjusted to $10^{8} \mathrm{CFU} / \mathrm{mL}$, and $1.2 \mathrm{~mL}$ sodium tetraborate was added into sulfuric acid in the resuspensions that were placed in ice bath and incubated for $5 \mathrm{~min}$ at $100^{\circ} \mathrm{C}$, and then kept on ice for $10 \mathrm{~min}$. A $20 \mu \mathrm{L}$ volume of $1.5 \mathrm{mg} / \mathrm{mL}$ m-hydroxyphenyl was then added and mixed. After incubating for $5 \mathrm{~min}$ at room temperature, the absorbance was measured at $590 \mathrm{~nm}$. The glucuronic acid content was determined by a standard curve of glucuronic acid and expressed as $\mu \mathrm{g} / 10^{8} \mathrm{CFU}$. The results were presented as the mean data from three independent experiments.

\section{Biofilm formation assay}

The biofilm formation assay was measured using the method described by Wilksch et al. [33]. Briefly, the clinical isolates were grown to logarithmic phase in LB broth and diluted at 1:100 ratio with fresh LB broth. Each dilution $(200 \mu \mathrm{L})$ was added to 96 -well polystyrene microtiter plates, with three duplicate wells per strain, as well as blank controls were set at the same time. The plates were then incubated at $37^{\circ} \mathrm{C}$ for $24 \mathrm{~h}$. The planktonic cells were removed, and the wells were washed thrice with sterile water, stained with $250 \mu \mathrm{L} 0.1 \%$ crystal violet for $10 \mathrm{~min}$ and then rinsed three times with sterile water. The stained biofilms were solubilized with $95 \%$ ethanol and quantified by measuring the $\mathrm{OD}_{600}$. Each sample was measured in triplicate, and the average of absorbance value was used for analysis.

\section{Serum killing test}

The serum bactericidal activity was measured as described by previous method [6]. The bacterial suspensions in the nutrient broth were collected during the logarithmic phase and adjusted to $10^{6} \mathrm{CFU} / \mathrm{mL}$. $25 \mu \mathrm{L}$ of bacterial suspension was added to $75 \mu \mathrm{L}$ of pooled human sera in the tube. Next, the tubes were shaken and incubated for 0,1 , 2, or $3 \mathrm{~h}$. An aliquot of each bacterial suspension was removed at the designated time point, diluted to corresponding fold by adding Mueller-Hinton broth, and then cultured to determine the number of viable bacteria after exposure to serum. The strain was considered serum resistant or serum sensitive according to the results that were expressed as the viable counts and graded, and each strain was tested at least three times.

\section{Infection model of Galleria mellonella larvae}

The model of G. mellonella larvae was established based on three PLA-causing MDR isolates (FK3068, FK3228, FK4603) and three typical hypervirulent strains (FK3112, FK3837, FK3914) that were randomly selected and standard strain ATCC 700603 to investigate the virulence and pathogenicity of the strains [34-35]. Serially diluted bacterial suspension of each strain $\left(10^{7}, 10^{6}, 10^{5}, 10^{4} \mathrm{CFU} / \mathrm{mL}\right)$ was prepared in advance. Eight larvae weighing $200 \mathrm{mg}-250 \mathrm{mg}$ were randomly selected for each strain and each concentration. A $10 \mu \mathrm{L}$ of bacterial suspension was injected into the last left proleg by using a $25 \mu \mathrm{L}$ Hamilton precision syringe. The larvae injected with $10 \mu \mathrm{L}$ phosphate-buffered saline were used as controls. After that, the insects were incubated at $37^{\circ} \mathrm{C}$ in the dark and observed after $24 \mathrm{~h}, 48 \mathrm{~h}, 72 \mathrm{~h}$ and $96 \mathrm{~h}$. The larvae were considered dead when they repeatedly fail to respond to physical stimuli. All experiments were done in triplicate.

\section{Polymerase chain reaction (PCR) for capsular serotypes and virulence genes}

Crude genomic DNA was extracted from PLA-causing K. pneumoniae isolates. Subsequently, the capsular serotype-specific genes (for serotypes of K1, K2, $\mathrm{K} 5, \mathrm{~K} 20, \mathrm{~K} 54$, and $\mathrm{K} 57$ ) and virulence genes (aerobactin, rmpA, iroN, kfuBC, wcaG, ybtA, magA, fimH, mrkD, uge, entB, and ureA) were amplified by PCR using specific primers as described previously (Table 2) [24,36-38]. In addition, the strains with these genes that were determined by PCR and DNA sequencing were selected as positive controls for subsequent PCR experiments.

\section{Multilocus sequence typing (MLST)}

Seven housekeeping genes of $K$. pneumoniae ( $g a p A, m d h$, $p h o E$, tonB, infB, pgi and $r p o B$ ) were amplified and sequenced to characterize the genotypes of PLA-causing isolates according to the provided protocols (http://bigsdb.pasteur.fr/klebsiella/klebsiella.html/). The alleles and sequence types were assigned according to the online database of the Pasteur Institute MLST for K. pneumoniae.

\section{Statistical analysis}

All statistical analyses were performed using SPSS 22.0 software (IBM, Armonk, NY, USA). Continuous variables were expressed as means \pm SD or median (25th - 75th percentile), whereas categorical variables were described as the number and percentage of subjects. Student's $t$ test or Mann-Whitney $U$ test was performed to evaluate the differences in continuous variables. Chi-square test or Fisher's exact test was applied to evaluate the differences in categorical variables. The mortality of G. mellonella was assessed by Kaplan-Meier analysis and log-rank test. Statistical tests were performed with a two-tailed significance level of 0.05 .

\section{List Of Abbreviations}

PLA: pyogenic liver abscess; MDR: multidrug resistant; MLST: multilocus sequence typing; cKP: classic K. pneumoniae, hvKP: hypervirulent $K$. pneumoniae; KP-PLA: K. pneumoniaeinduced pyogenic liver abscess; MICs: minimum inhibitory concentrations; PCR: polymerase chain reaction; STs: sequence types 


\section{Declarations}

\section{Ethics approval and consent to participate}

This study has been designed in accordance with the Declaration of Helsinki (2013) (https://www.wma.net/policies-post/wma-declaration-of-helsinki-ethicalprinciples-for-medical-research-involving-human-subjects/ $\$ and been approved by the Ethics Committee in Clinical Research of the First Affiliated Hospital of Wenzhou Medical University (No.2020-070).

\section{Consent for publication}

Not applicable.

\section{Availability of data and materials}

The datasets used and analyzed during the study are available from the corresponding author on reasonable request.

\section{Competing interests}

The authors declare that they have no competing interests.

\section{Funding}

This work was supported by research grants from the National Natural Science Foundation of China (No.81741059 and 81971986) and the Health Department of Zhejiang Province of the People's Republic of China (No. 2019KY098)

\section{Author Contributions}

All authors contributed to data analysis, drafting and revising the article, gave final approval of the version to be published,and agree to be accountable for all aspects of the work.

\section{Acknowledgement}

We thank the National Natural Science Foundation of China (No.81741059 and 81971986) and the Health Department of Zhejiang Province of the People's Republic of China (No. 2019KY098) for providing financial funding.

\section{References}

1. Russo TA, Marr CM: Hypervirulent Klebsiella pneumoniae. Clin Microbiol Rev 2019, 32(3).

2. Siu LK, Yeh KM, Lin JC, Fung CP, Chang FY: Klebsiella pneumoniae liver abscess: a new invasive syndrome. Lancet Infect Dis 2012, 12(11):881-887.

3. Liu YM, Li BB, Zhang YY, Zhang W, Shen H, Li H, Cao B: Clinical and molecular characteristics of emerging hypervirulent Klebsiella pneumoniae bloodstream infections in mainland China. Antimicrob Agents Chemother 2014, 58(9):5379-5385.

4. Tsai FC, Huang YT, Chang LY, Wang JT: Pyogenic liver abscess as endemic disease, Taiwan. Emerg Infect Dis 2008, 14(10):1592-1600.

5. Shon AS, Bajwa RP, Russo TA: Hypervirulent (hypermucoviscous) Klebsiella pneumoniae: a new and dangerous breed. Virulence 2013, 4(2):107-118.

6. Siu LK, Fung CP, Chang FY, Lee N, Yeh KM, Koh TH, Ip M: Molecular typing and virulence analysis of serotype K1 Klebsiella pneumoniae strains isolated from liver abscess patients and stool samples from noninfectious subjects in Hong Kong, Singapore, and Taiwan. J Clin Microbio/ 2011, 49(11):3761-3765.

7. Chen J, Zhang M, Chen J, Ning Y, Cai X, Zhang L, Xu H, Guo J: Cryptogenic and non-cryptogenic liver abscess: A retrospective analysis of 178 cases revealed distinct characteristics. J Int Med Res 2018, 46(9):3824-3836.

8. Kong H, Yu F, Zhang W, Li X: Clinical and microbiological characteristics of pyogenic liver abscess in a tertiary hospital in East China. Medicine (Baltimore) 2017, 96(37):e8050.

9. Gu D, Dong N, Zheng Z, Lin D, Huang M, Wang L, Chan EW, Shu L, Yu J, Zhang R et al: A fatal outbreak of ST11 carbapenem-resistant hypervirulent Klebsiella pneumoniae in a Chinese hospital: a molecular epidemiological study. Lancet Infect Dis 2018, 18(1):37-46.

10.Yang X, Wai-Chi Chan E, Zhang R, Chen S: A conjugative plasmid that augments virulence in Klebsiella pneumoniae. Nat Microbiol $2019,4(12): 2039-2043$.

11.Zhang S, Zhang X, Wu Q, Zheng X, Dong G, Fang R, Zhang Y, Cao J, Zhou T: Clinical, microbiological, and molecular epidemiological characteristics of Klebsiella pneumoniaeinduced pyogenic liver abscess in southeastern China. Antimicrob Resist Infect Control 2019, 8:166.

12. Jun JB: Klebsiella pneumoniae Liver Abscess. Infect Chemother 2018, 50(3):210-218.

13.Liu Y, Liu PP, Wang LH, Wei DD, Wan LG, Zhang W: Capsular Polysaccharide Types and Virulence-Related Traits of Epidemic KPC-Producing Klebsiella pneumoniae Isolates in a Chinese University Hospital. Microb Drug Resist 2017, 23(7):901-907. 
14. Ye M, Tu J, Jiang J, Bi Y, You W, Zhang Y, Ren J, Zhu T, Cao Z, Yu Z et al: Clinical and Genomic Analysis of Liver Abscess-Causing Klebsiella pneumoniae Identifies New Liver Abscess-Associated Virulence Genes. Front Cell Infect Microbiol 2016, 6:165.

15.Lee CR, Lee JH, Park KS, Jeon JH, Kim YB, Cha CJ, Jeong BC, Lee SH: Antimicrobial Resistance of Hypervirulent Klebsiella pneumoniae: Epidemiology, Hypervirulence-Associated Determinants, and Resistance Mechanisms. Front Cell Infect Microbiol 2017, 7:483.

16.Su SC, Siu LK, Ma L, Yeh KM, Fung CP, Lin JC, Chang FY: Community-acquired liver abscess caused by serotype K1 Klebsiella pneumoniae with CTX-M-15type extended-spectrum beta-lactamase. Antimicrob Agents Chemother 2008, 52(2):804-805.

17. Fung CP, Chang FY, Lin JC, Ho DM, Chen CT, Chen JH, Yeh KM, Chen TL, Lin YT, Siu LK: Immune response and pathophysiological features of Klebsiella pneumoniae liver abscesses in an animal model. Lab Invest 2011, 91(7):1029-1039.

18.Elliott AG, Ganesamoorthy D, Coin L et al. Complete Genome Sequence of Klebsiella quasipneumoniae subsp.similipneumoniae Strain ATCC 700603.Genome Announc 2016, 26;4(3):e00438-16.

19.McLaughlin MM, Advincula MR, Malczynski M et al. Quantifying the clinical virulence of Klebsiella pneumoniae producing carbapenemase Klebsiella pneumoniae with a Galleria mellonella model and a pilot study to translate to patient outcomes. BMC Infect Dis 2014, 14: 31.

20. Podschun R, Ullmann U: Klebsiella spp. as nosocomial pathogens: epidemiology, taxonomy, typing methods, and pathogenicity factors. Clin Microbiol $\operatorname{Rev} 1998,11(4): 589-603$.

21.Yu WL, Ko WC, Cheng KC, Lee CC, Lai CC, Chuang YC: Comparison of prevalence of virulence factors for Klebsiella pneumoniae liver abscesses between isolates with capsular K1/K2 and non-K1/K2 serotypes. Diagn Microbiol Infect Dis 2008, 62(1):1-6.

22. Yu WL, Ko WC, Cheng KC, Lee HC, Ke DS, Lee CC, Fung CP, Chuang YC: Association between rmpA and magA genes and clinical syndromes caused by Klebsiella pneumoniae in Taiwan. Clin Infect Dis 2006, 42(10):1351-1358.

23. Paczosa MK, Mecsas J: Klebsiella pneumoniae: Going on the Offense with a Strong Defense. Microbiol Mol Biol Rev 2016, 80(3):629-661.

24. Zhang S, Yang G, Ye Q, Wu Q, Zhang J, Huang Y: Phenotypic and Genotypic Characterization of Klebsiella pneumoniae Isolated From Retail Foods in China. Front Microbiol 2018, 9:289.

25.Yeh KM, Lin JC, Yin FY, Fung CP, Hung HC, Siu LK, Chang FY: Revisiting the importance of virulence determinant magA and its surrounding genes in Klebsiella pneumoniae causing pyogenic liver abscesses: exact role in serotype K1 capsule formation. J Infect Dis 2010, 201(8):1259-1267.

26. Struve C, Bojer M, Krogfelt KA: Identification of a conserved chromosomal region encoding Klebsiella pneumoniae type 1 and type 3 fimbriae and assessment of the role of fimbriae in pathogenicity. Infect Immun 2009, 77(11):5016-5024.

27. Lam MMC, Wyres KL, Duchene S, Wick RR, Judd LM, Gan YH, Hoh CH, Archuleta S, Molton JS, Kalimuddin S et al: Population genomics of hypervirulent Klebsiella pneumoniae clonal-group 23 reveals early emergence and rapid global dissemination. Nat Commun 2018, 9(1):2703.

28. Foo NP, Chen KT, Lin HJ, Guo HR: Characteristics of pyogenic liver abscess patients with and without diabetes mellitus. Am J Gastroentero/ 2010, 105(2):328-335.

29.Magiorakos AP, Srinivasan A, Carey RB, Carmeli Y, Falagas ME, Giske CG, Harbarth S, Hindler JF, Kahlmeter G, Olsson-Liljequist B et al: Multidrug-resistant, extensively drug-resistant and pandrug-resistant bacteria: an international expert proposal for interim standard definitions for acquired resistance. Clin Microbiol Infect 2012, 18(3):268-281.

30. Russo TA, Olson R, Fang CT, Stoesser N, Miller M, MacDonald U, Hutson A, Barker JH, La Hoz RM, Johnson JR: Identification of Biomarkers for Differentiation of Hypervirulent Klebsiella pneumoniae from Classical K. pneumoniae. J Clin Microbiol 2018, 56(9).

31.Palacios M, Broberg CA, Walker KA, Miller VL: A Serendipitous Mutation Reveals the Severe Virulence Defect of a Klebsiella pneumoniae fepB Mutant. mSphere 2017, 2(4).

32.Filisetti-Cozzi TM, Carpita NC: Measurement of uronic acids without interference from neutral sugars. Anal Biochem 1991, 197(1):157-162.

33.Wilksch JJ, Yang J, Clements A et al. MrkH, a novel c-di-GMP-dependent transcriptional activator, controls Klebsiella pneumoniae biofilm formation by regulating type 3 fimbriae expression. PLoS Pathog 2011, 7(8): e1002204.

34.Zhang X, Zhao Y, Wu Q, Lin J, Fang R, Bi W, Dong G, Li J, Zhang Y, Cao J et al: Zebrafish and Galleria mellonella: Models to Identify the Subsequent Infection and Evaluate the Immunological Differences in Different Klebsiella pneumoniae Intestinal Colonization Strains. Front Microbiol $2019,10: 2750$.

35.Insua JL, Llobet E, Moranta D et al. Modeling Klebsiella pneumoniae pathogenesis by infection of the wax moth Galleria mellonella. Infect Immun. 2013; 81(10): 3552-65.

36. Candan ED, Aksoz N: Klebsiella pneumoniae: characteristics of carbapenem resistance and virulence factors. Acta Biochim Pol2015, 62(4):867-874. 
37. Luo Y, Wang Y, Ye L, Yang J: Molecular epidemiology and virulence factors of pyogenic liver abscess causing Klebsiella pneumoniae in China. Clin Microbiol Infect 2014, 20(11):0818-824.

38. Wasfi R, Elkhatib WF, Ashour HM: Molecular typing and virulence analysis of multidrug resistant Klebsiella pneumoniae clinical isolates recovered from Egyptian hospitals. Sci Rep 2016, 6:38929.

\section{Tables}

Table 1. The MICs of PLA-causing multidrug resistant strains and control strains against antimicrobial agents 


\begin{tabular}{|c|c|c|c|c|c|c|c|c|c|c|c|c|c|c|c|}
\hline \multirow{2}{*}{$\begin{array}{l}\text { Strain } \\
\text { numbers }\end{array}$} & \multirow{2}{*}{$\begin{array}{l}\text { Isolate } \\
\text { date }\end{array}$} & \multirow{2}{*}{$\begin{array}{l}\text { Isolate } \\
\text { type }\end{array}$} & \multicolumn{13}{|c|}{ MICs $(\mu \mathrm{g} / \mathrm{mL}, \mathrm{mg} / \mathrm{L})$} \\
\hline & & & AMP & ATM & CRO & CAZ & FEP & IPM & CIP & LEV & GEN & TOB & SXT & NIT & $\mathrm{COL}$ \\
\hline FK3038 & $\begin{array}{l}\text { May 17, } \\
2016\end{array}$ & $\begin{array}{l}\text { drainage } \\
\text { fluid }\end{array}$ & $>64$ & 2 & $>64$ & 16 & 4 & $>64$ & $>8$ & 1 & 2 & 1 & $<0.25 / 4.75$ & 128 & 0.5 \\
\hline FK3044 & $\begin{array}{l}\text { May 19, } \\
2016\end{array}$ & pus & 32 & 16 & $>64$ & $>64$ & 32 & 1 & 0.03 & 2 & 0.125 & 4 & $>16 / 304$ & 128 & 1 \\
\hline FK3068 & $\begin{array}{l}\text { May 26, } \\
2016\end{array}$ & pus & 32 & 16 & $>64$ & 16 & 32 & 0.5 & 0.03 & 2 & 0.5 & 4 & $>16 / 304$ & 128 & 0.25 \\
\hline FK3228 & $\begin{array}{l}\text { July } 26 \text {, } \\
2016\end{array}$ & $\begin{array}{l}\text { drainage } \\
\text { fluid }\end{array}$ & 32 & $>64$ & $>64$ & $>64$ & $>32$ & 4 & 0.06 & $>16$ & 0.125 & 4 & $>16 / 304$ & 256 & 16 \\
\hline FK3347 & $\begin{array}{l}\text { September } \\
08,2016\end{array}$ & blood & 32 & 2 & 4 & 8 & $>32$ & 1 & 0.5 & $>16$ & 0.5 & 4 & $>16 / 304$ & 256 & 0.25 \\
\hline FK3518 & $\begin{array}{l}\text { November } \\
09,2016\end{array}$ & pus & $>64$ & 2 & $>64$ & 16 & 2 & 1 & $>8$ & 1 & 0.5 & 0.5 & $1 / 19$ & 256 & 0.25 \\
\hline FK3521 & $\begin{array}{l}\text { November } \\
10,2016\end{array}$ & pus & $>64$ & 2 & $>64$ & 16 & 16 & 1 & $>8$ & 4 & 0.5 & $<0.25$ & $<0.25 / 4.75$ & 128 & 0.25 \\
\hline FK3599 & $\begin{array}{l}\text { December } \\
13,2016\end{array}$ & pus & 32 & 2 & 4 & 2 & 0.25 & 4 & 8 & 1 & 0.5 & $<0.25$ & $<0.25 / 4.75$ & 256 & 0.25 \\
\hline$\underline{F K 4176}$ & $\begin{array}{l}\text { July 13, } \\
2017\end{array}$ & $\begin{array}{l}\text { drainage } \\
\text { fluid }\end{array}$ & $>64$ & 2 & 64 & 8 & 16 & 0.5 & 2 & 4 & 16 & 0.5 & $<0.25 / 4.75$ & 128 & 0.5 \\
\hline FK4276 & $\begin{array}{l}\text { August } \\
14,2017\end{array}$ & $\begin{array}{l}\text { drainage } \\
\text { fluid }\end{array}$ & $>64$ & 2 & $>64$ & 32 & 16 & 1 & 4 & 1 & 16 & $<0.25$ & $<0.25 / 4.75$ & 32 & 1 \\
\hline FK4603 & $\begin{array}{l}\text { November } \\
13,2017\end{array}$ & blood & 32 & $>64$ & 64 & 64 & $>32$ & 1 & 4 & $>16$ & 0.25 & 2 & $>16 / 304$ & 256 & 0.25 \\
\hline FK4737 & $\begin{array}{l}\text { December } \\
26,2017\end{array}$ & pus & 32 & 32 & $>64$ & 32 & 8 & 2 & 4 & 4 & 0.5 & 4 & $>16 / 304$ & 128 & 0.125 \\
\hline FK3112 & $\begin{array}{l}\text { June } \\
13.2016\end{array}$ & pus & 32 & 1 & $<0.03$ & 0.125 & $<0.03$ & 0.125 & 0.03 & 2 & 0.25 & 1 & $0.5 / 9.5$ & 32 & 0.5 \\
\hline FK3262 & $\begin{array}{l}\text { August } \\
08,2016\end{array}$ & pus & 32 & 0.5 & $<0.03$ & 0.125 & $<0.03$ & 0.125 & 0.03 & 0.06 & 0.25 & 0.5 & $0.5 / 9.5$ & 32 & 0.25 \\
\hline FK3645 & $\begin{array}{l}\text { January } \\
10,2017\end{array}$ & pus & $>64$ & 0.5 & $<0.03$ & 0.125 & $<0.03$ & 0.125 & 0.03 & 0.06 & 0.5 & 0.5 & $<0.25 / 4.75$ & 32 & 1 \\
\hline FK3698 & $\begin{array}{l}\text { February } \\
08,2017\end{array}$ & pus & 32 & 0.5 & $<0.03$ & 0.25 & $<0.03$ & 0.25 & 0.03 & 0.06 & 1 & $<0.25$ & $<0.25 / 4.75$ & 16 & 0.25 \\
\hline FK3736 & $\begin{array}{l}\text { February } \\
20,2017\end{array}$ & pus & 32 & 0.25 & $<0.03$ & 0.125 & $<0.03$ & 0.25 & 0.03 & 1 & 0.5 & 0.5 & $<0.25 / 4.75$ & 16 & 0.5 \\
\hline FK3818 & $\begin{array}{l}\text { March } \\
21.2017\end{array}$ & pus & 32 & 1 & $<0.03$ & 0.25 & $<0.03$ & 0.25 & 0.5 & 0.25 & 0.25 & 0.5 & $<0.25 / 4.75$ & 32 & 1 \\
\hline FK3837 & $\begin{array}{l}\text { March } \\
24.2017\end{array}$ & pus & 32 & 1 & $<0.03$ & 0.25 & $<0.03$ & 0.25 & 0.03 & 0.06 & 0.25 & 0.5 & $<0.25 / 4.75$ & 32 & 0.25 \\
\hline FK3914 & $\begin{array}{l}\text { April } \\
21,2017\end{array}$ & pus & 32 & 0.5 & $<0.03$ & 0.125 & $<0.03$ & 0.25 & 0.03 & 0.06 & 0.25 & 0.5 & $<0.25 / 4.75$ & 32 & 0.25 \\
\hline FK3953 & $\begin{array}{l}\text { May 05, } \\
2017\end{array}$ & pus & 32 & 0.5 & $<0.03$ & 0.125 & $<0.03$ & 0.25 & 0.03 & 0.5 & 0.5 & 0.5 & $<0.25 / 4.75$ & 32 & 0.5 \\
\hline FK3992 & $\begin{array}{l}\text { May 17, } \\
2017\end{array}$ & $\begin{array}{l}\text { drainage } \\
\text { fluid }\end{array}$ & 32 & 0.5 & $<0.03$ & 0.125 & $<0.03$ & 0.25 & 0.008 & 0.25 & 0.25 & 0.5 & $<0.25 / 4.75$ & 32 & 0.5 \\
\hline FK4081 & $\begin{array}{l}\text { June } \\
15.2017\end{array}$ & $\begin{array}{l}\text { drainage } \\
\text { fluid }\end{array}$ & $>64$ & 0.5 & $<0.03$ & 0.125 & $<0.03$ & 0.125 & 0.03 & 0.06 & 0.25 & 0.5 & $<0.25 / 4.75$ & 32 & 1 \\
\hline FK4578 & $\begin{array}{l}\text { November } \\
06,2017\end{array}$ & pus & $>64$ & 0.5 & $<0.03$ & 0.125 & $<0.03$ & 0.25 & 0.03 & 0.06 & 0.25 & $<0.25$ & $<0.25 / 4.75$ & 16 & 1 \\
\hline
\end{tabular}

MICs minimum inhibitory concentrations, PLA pyogenic liver abscess, AMP ampicillin, ATM aztreonam, CRO ceftriaxone, CAZ ceftazidime, FEP cefepime, IPM imipenem, CIP ciprofloxacin, LVX levofloxacin, GEN gentamicin, TOB tobramycin, SXT sulfamethoxazole/trimethoprim, NIT nitrofurantoin, COL colistin.

Strain numbers were underlined: multidrug resistant strains; strain numbers were bolded: hypervirulent control strains. gray shading: resistance, white shading: intermediate or susceptible 
Table 2. List of oligonucleotide primers used in amplification of capsular serotypes and virulence genes

\begin{tabular}{|c|c|c|c|}
\hline Gene & Primer Sequence $\left(5^{\prime}-3^{\prime}\right)$ & $\operatorname{Tm}\left({ }^{\circ} \mathrm{C}\right)$ & Amplicon size (bp) \\
\hline \multirow[t]{2}{*}{$\operatorname{magA}$} & F: GGTGCTCTTTACATCATTGC & \multirow[t]{2}{*}{58} & \multirow[t]{2}{*}{1283} \\
\hline & R: GCAATGGCCATTTGCGTTAG & & \\
\hline \multirow[t]{2}{*}{ aerobactin } & F: GCATAGGCGGATACGAACAT & \multirow[t]{2}{*}{58} & \multirow[t]{2}{*}{556} \\
\hline & R: CACAGGGCAATTGCTTACCT & & \\
\hline \multirow[t]{2}{*}{$\operatorname{iroN}$} & F: AAGTCAAAGCAGGGGTTGCCCG & \multirow[t]{2}{*}{58} & \multirow[t]{2}{*}{665} \\
\hline & R: GACGCCGACATTAAGACGCAG & & \\
\hline \multirow[t]{2}{*}{$k f u B C$} & F: GAAGTGACGCTGTTTCTGGC & \multirow[t]{2}{*}{58} & \multirow[t]{2}{*}{979} \\
\hline & R: TTTCGTGTGGCCAGTGACTC & & \\
\hline \multirow[t]{2}{*}{$r m p A$} & F: ACTGGGCTACCTCTGCTTCA & \multirow[t]{2}{*}{58} & \multirow[t]{2}{*}{516} \\
\hline & R: CTTGCATGAGCCATCTTTCA & & \\
\hline \multirow[t]{2}{*}{$w c a G$} & F: GGTTGGKTCAGCAATCGTA & \multirow[t]{2}{*}{58} & \multirow[t]{2}{*}{169} \\
\hline & R: ACTATTCCGCCAACTTTTGC & & \\
\hline \multirow[t]{2}{*}{$y b t A$} & F: ATGACGGAGTCACCGCAAAC & \multirow[t]{2}{*}{55} & \multirow[t]{2}{*}{960} \\
\hline & R: TTACATCACGCGTTTAAAGG & & \\
\hline \multirow[t]{2}{*}{ ureA } & F: GCTGACTTAAGAGAACGTTATG & \multirow[t]{2}{*}{58} & \multirow[t]{2}{*}{337} \\
\hline & R: GATCATGGCGCTACCT(C/T)A & & \\
\hline \multirow[t]{2}{*}{ uge } & F: TCTTCACGCCTTCСTTCACT & \multirow[t]{2}{*}{53} & \multirow[t]{2}{*}{534} \\
\hline & R: GATCATCCGGTCTCCCTGTA & & \\
\hline \multirow[t]{2}{*}{ entB } & F: АTTTCCTCAACTTCTGGGGC & \multirow[t]{2}{*}{56} & 371 \\
\hline & R: AGCATCGGTGGCGGTGGTCA & & \\
\hline $\mathrm{fimH}$ & F: TGCTGCTGGGCTGGTCGATG & 62 & 688 \\
\hline & R: GGGAGGGTGACGGTGACATC & & \\
\hline$m r k D$ & F: CCACСААСТАTTСССТCGAА & 43 & 226 \\
\hline & R: ATGGAACCCACATCGACATT & & \\
\hline $\mathrm{K} 1$ & F: GGTGCTCTTTACATCATTGC & 54 & 1283 \\
\hline & R: GCAATGGCCATTTGCGTTAG & & \\
\hline $\mathrm{K} 2$ & F: GGATTATGACAGCCTCTCCT & 58 & 641 \\
\hline & R: CGACTTGGTCCCAACAGTTT & & \\
\hline K5 & F: TGGTAGTGATGCTCGCGA & 58 & 280 \\
\hline & R: CCTGAACCCACCCCAATC & & \\
\hline K20 & F: CGGTGCTACAGTGCATCATT & 58 & 741 \\
\hline & R: GTTATACGATGCTCAGTCGC & & \\
\hline K54 & F: CATTAGCTCAGTGGTTGGCT & 58 & 881 \\
\hline & R: GCTTGACAAACACCATAGCAG & & \\
\hline K57 & F: CTCAGGGCTAGAAGTGTCAT & 58 & 1037 \\
\hline & R: CTCAGGGCTAGAAGTGTCAT & & \\
\hline
\end{tabular}

Figures 


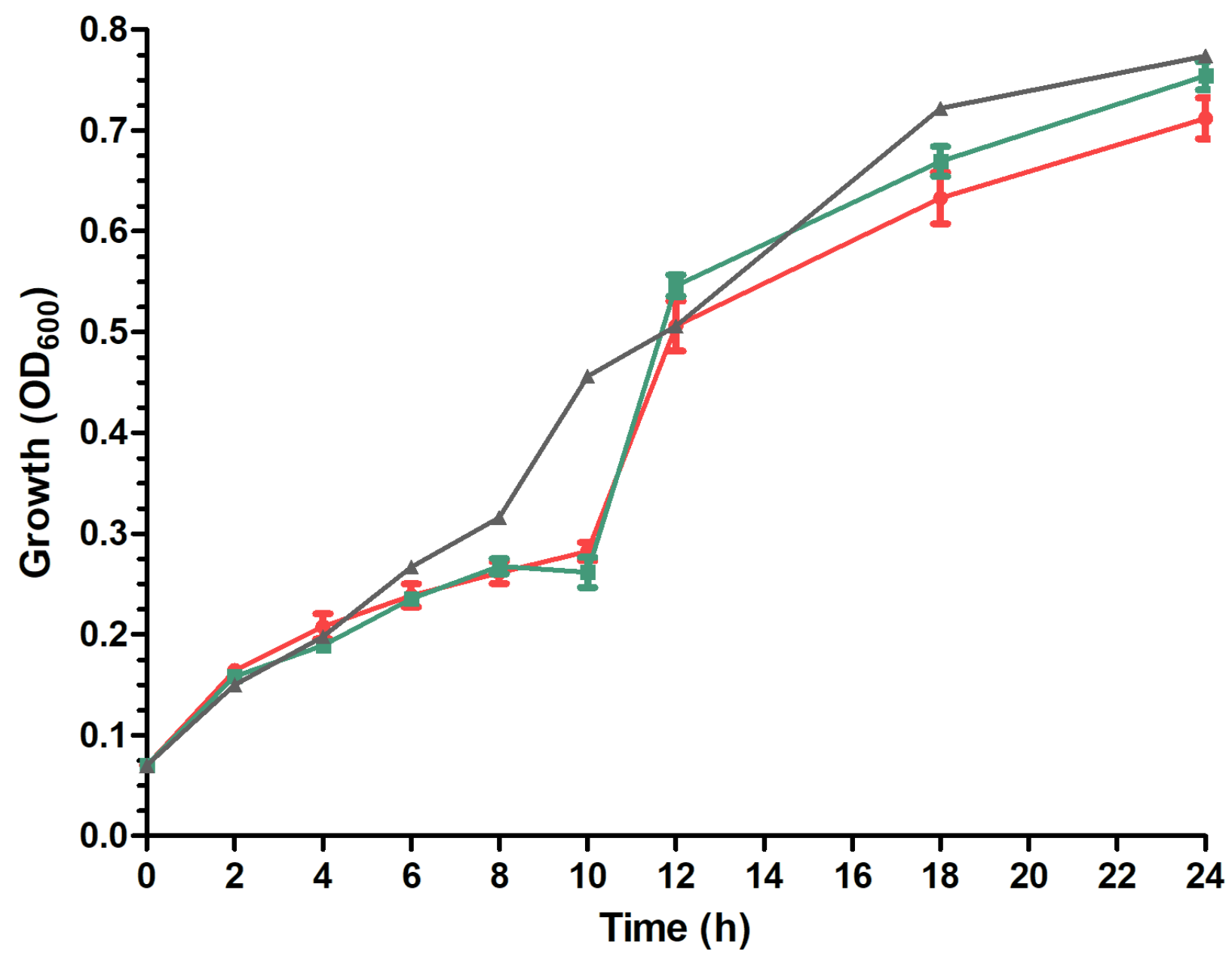

Figure 1

Growth curves of PLA-causing MDR K. pneumoniae strains (red circles, $n=12$ ) were comparable to that of typical hypervirulent strains (green squares, $n=12$ ) and standard strain ATCC 700603 (grey triangles, $n=1$ ). Data is presented as means $\pm S D$, with $N=3$. Statistical analysis was performed using Student's $t$ test, There was no significant difference between MDR strains and control strains (typical hypervirulent strains and standard strain ATCC 700603 ). P > 0.05 . 


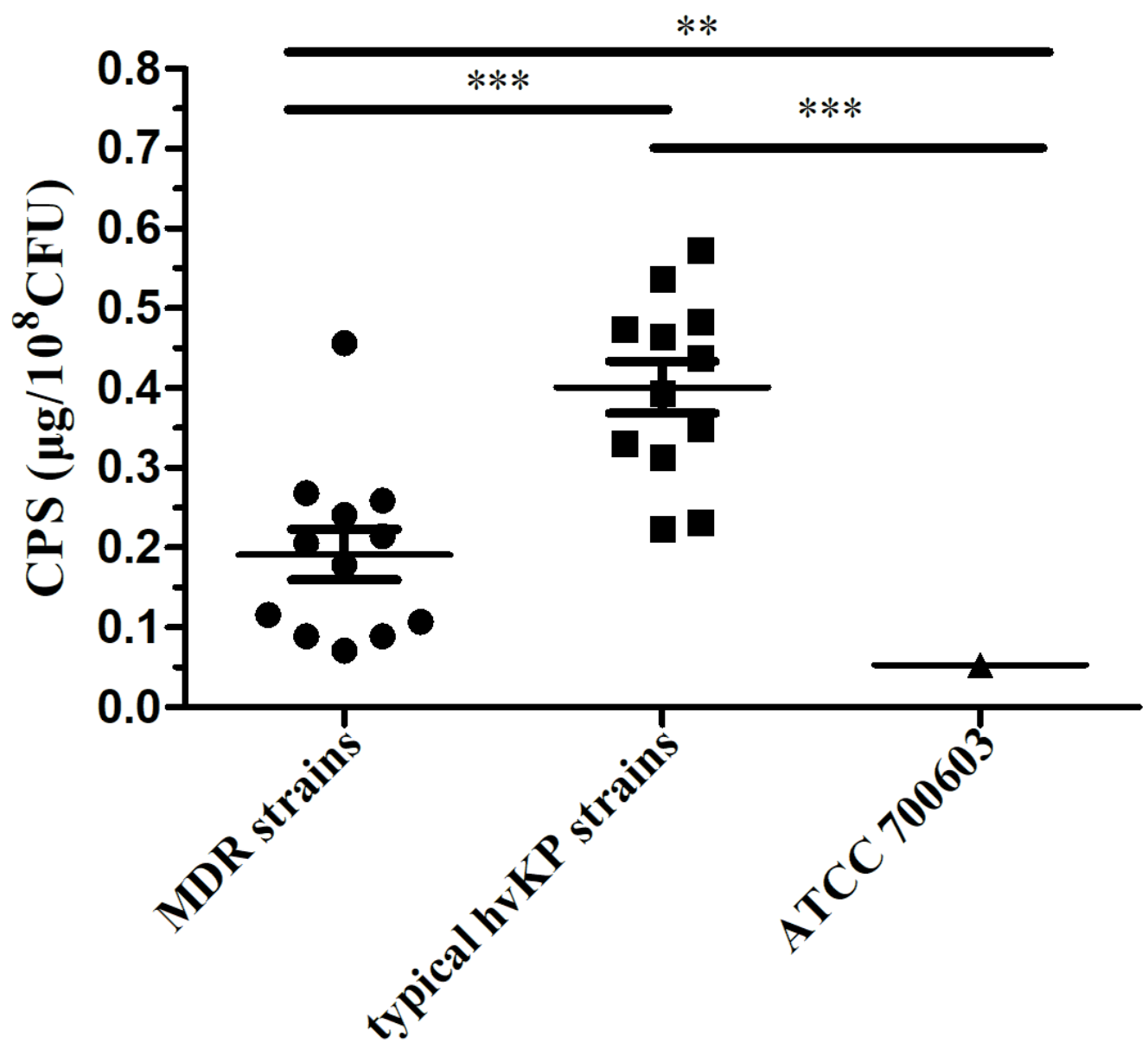

Figure 2

Capsular quantification of PLA-causing MDR K. pneumoniae strains (black circles, $n=12$ ) were comparable to that of typical hypervirulent strains (black squares, $n=12$ ) and standard strain ATCC 700603 (black triangles, $n=1$ ). Data is presented as means $\pm S D$, with $N=3$. Statistical analysis was performed using Student's t test. *, $\mathrm{P}<0.05$; $* *, \mathrm{P}<0.01$; ***, $\mathrm{P}<0.001$. 


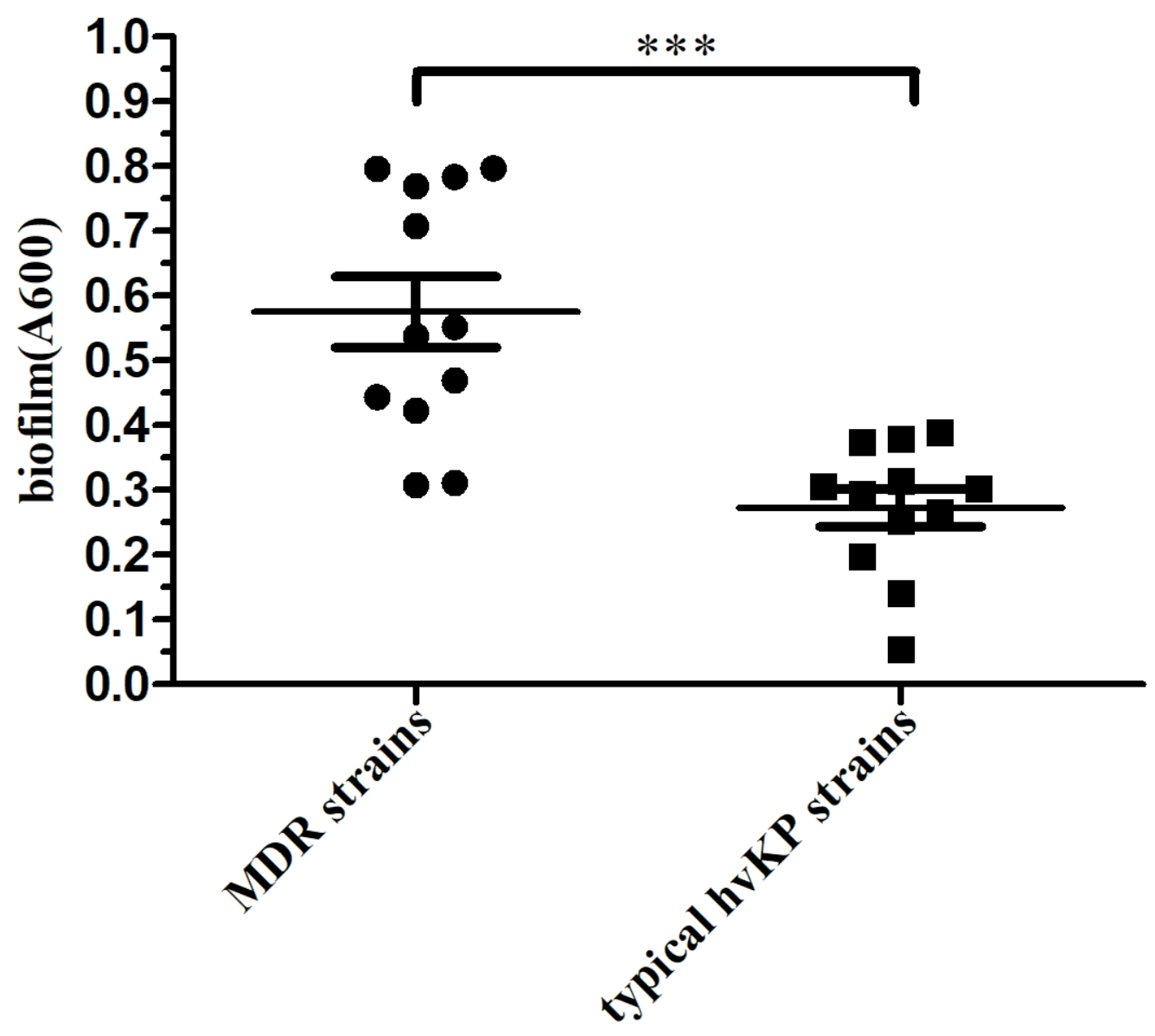

Figure 3

Biofilm formation ability of PLA-causing MDR K. pneumoniae strains (black circles, $n=12$ ) were comparable to that of typical hypervirulent strains (black squares, $n=12$ ). Data is presented as means $\pm S D$, with $N=3$. Statistical analysis was performed using Student's $t$ test. *, $P<0.05 ; * \star, P<0.01 ; * \star \star, P<0.001$. 


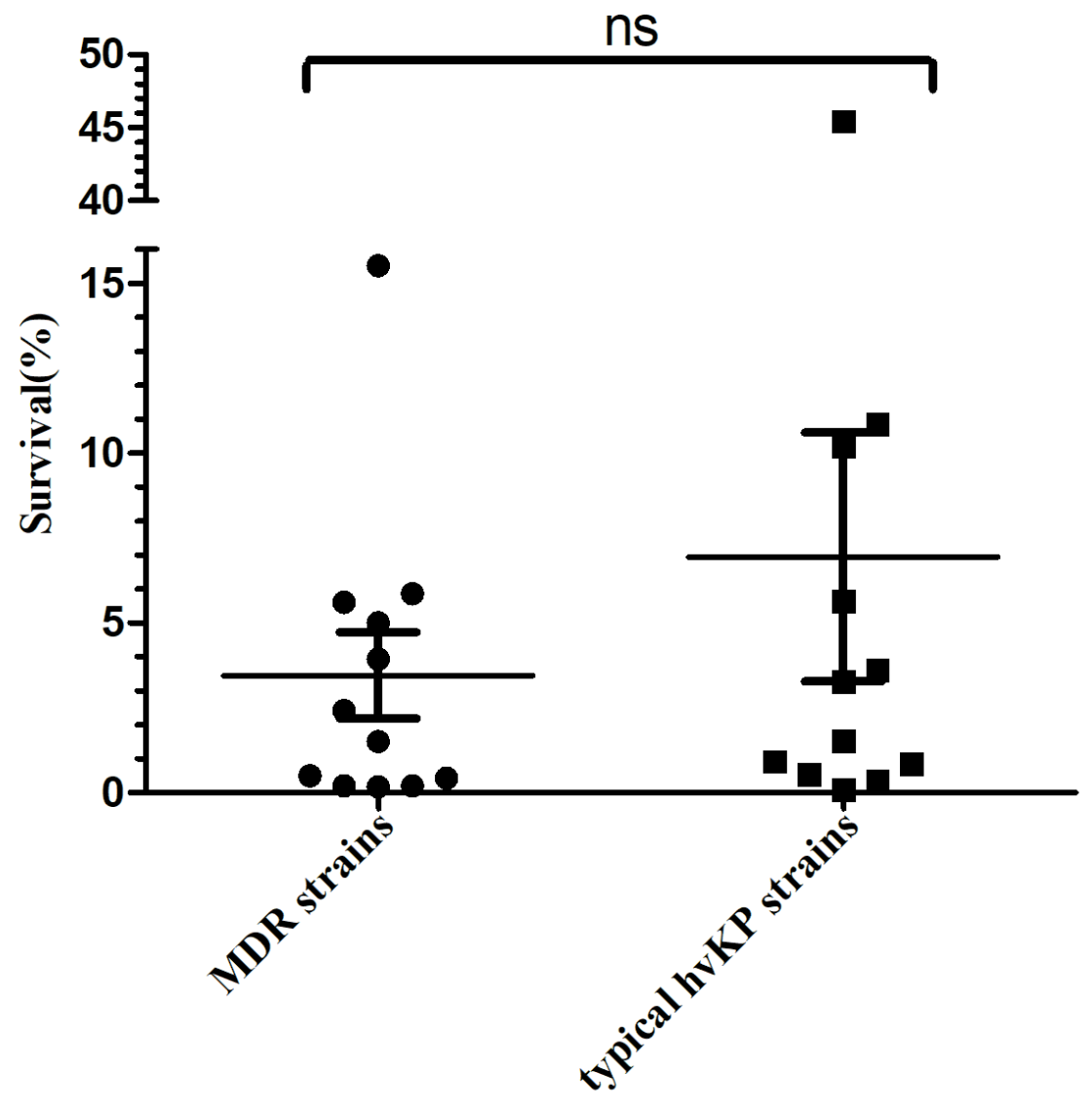

Figure 4

Serum bactericidal activity of PLA-causing MDR K. pneumoniae strains (black circles, $n=12$ ) were comparable to that of typical hypervirulent strains (black squares, $n=12$ ). Data is presented as means $\pm S D$, with $N=3$. Statistical analysis was performed using Student's t test. $n s$, not significant. 

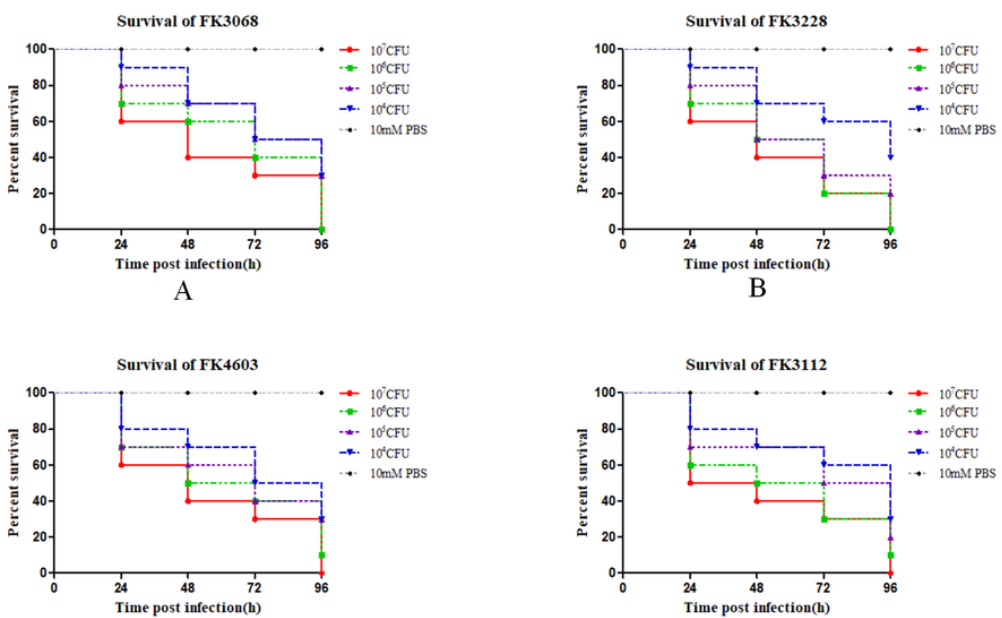

$\mathrm{C}$
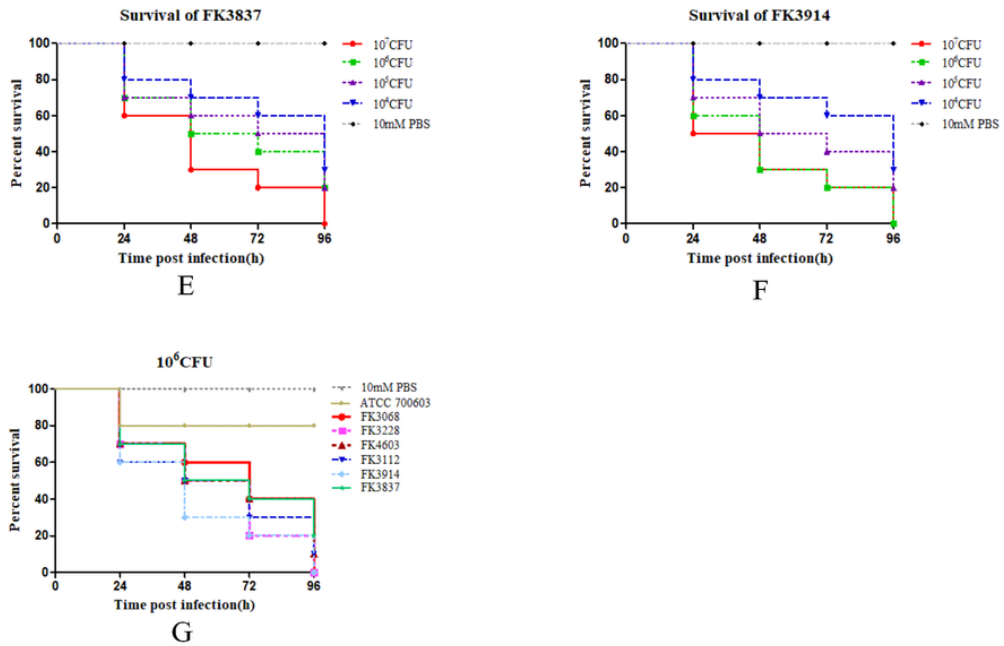

Figure 5

Survival curves of K. pneumoniae infection model of Galleria mellonella larvae. (A) FK3068, MDR strain; (B) FK3228, MDR strain; (C) FK4603, MDR strain; (D) FK3112, typical hypervirulent strain; (E) FK3837, typical hypervirulent strain; (F) FK3914, typical hypervirulent strain; (G) 106 CFU/mL bacterial suspensions of MDR strains (FK3068, FK3228, FK4603), typical hypervirulent strains (FK3112, FK3837, FK3914), a standard strain ATCC 700603, and a blank control PBS. Data is presented as percentage, with $\mathrm{N}=3$. Statistical analysis was performed using Kaplan-Meier analysis and log-rank test. 


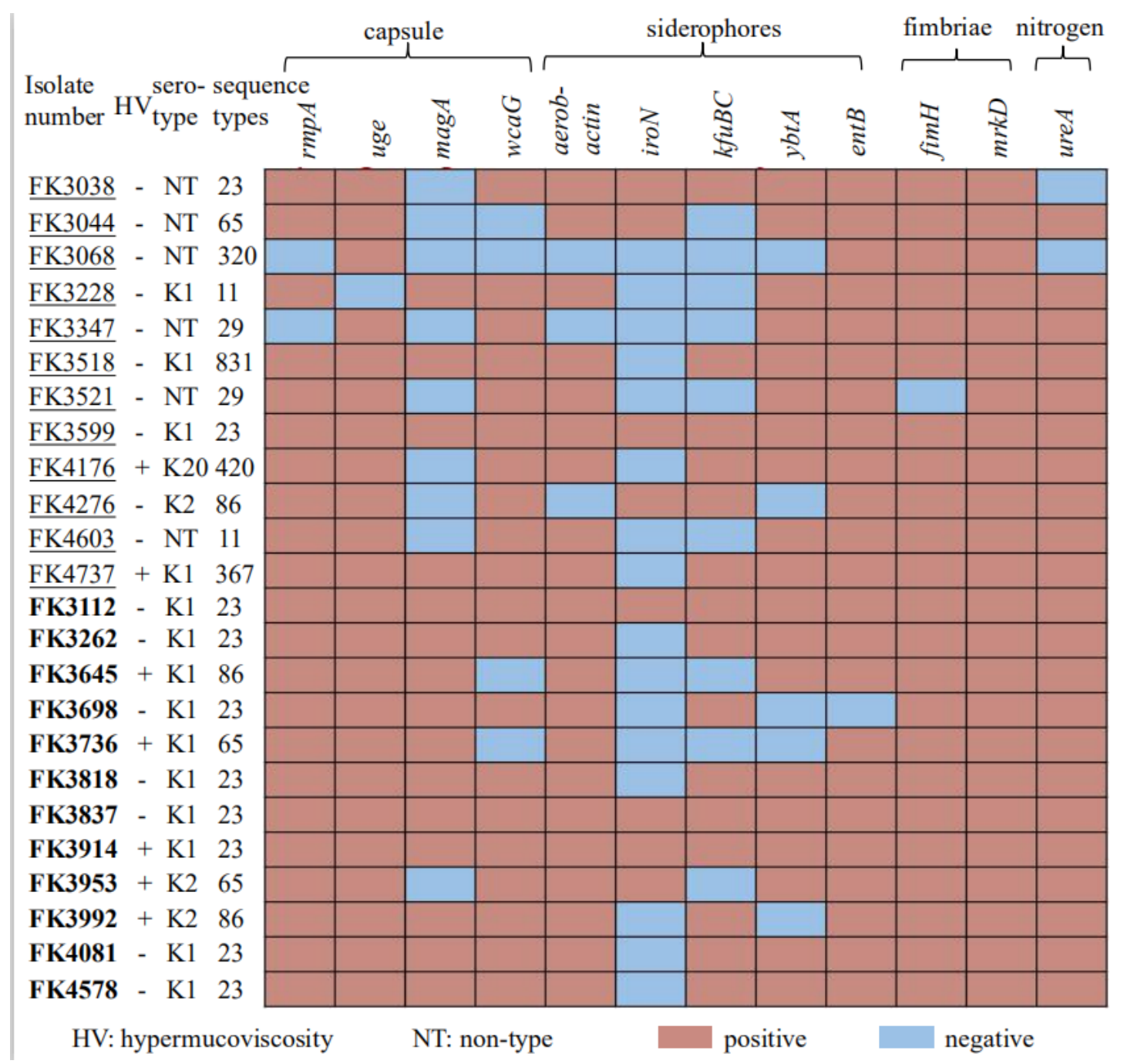

\section{Figure 6}

Genotype map of PLA-causing MDR and typical hypervirulent strains. Strain numbers were underlined: multidrug resistant strains; strain numbers were bolded: typical hypervirulent strains.

\section{Supplementary Files}

This is a list of supplementary files associated with this preprint. Click to download.

- Tables1.docx 